\title{
Comparison of Acoustic Respiration Rate, Impedance Pneumography and Capnometry Monitors for Respiration Rate Accuracy and Apnea Detection during GI Endoscopy Anesthesia
}

\author{
Basavana Gouda Goudra ${ }^{1}$, Lakshmi C. Penugonda ${ }^{2}$, Rebecca M. Speck ${ }^{1,3}$ Ashish C. Sinha $^{4}$ \\ ${ }^{1}$ Department of Anesthesiology and Critical Care, Perelman School of Medicine, Hospital of the University of Pennsylvania, Phila- \\ delphia, USA; ${ }^{2}$ Department of Surgery, Perelman School of Medicine, Hospital of the University of Pennsylvania, Philadelphia, \\ USA; ${ }^{3}$ Department of Biostatistics and Epidemiology, Perelman School of Medicine, Hospital of the University of Pennsylvania, \\ Philadelphia, USA; ${ }^{4}$ Department of Anesthesiology and Perioperative Medicine, Drexel University College of Medicine, Philadel- \\ phia, USA. \\ Email: goudrab@uphs.upenn.edu
}

Received January $7^{\text {th }}, 2013$; revised February $10^{\text {th }}, 2013$; accepted February $28^{\text {th }}, 2013$

\begin{abstract}
Study Objective: To assess the accuracy of respiration rate measurements and the ability to detect apnea by capnometry, impedance pneumography and a new method, acoustic respiration rate monitoring, in anesthetized patients undergoing gastrointestinal endoscopy procedures. Design: Prospective observational study. Setting: Endoscopy procedures laboratory. Patients: 98 patients scheduled for upper gastrointestinal endoscopy with propofol-based anesthesia. Interventions: Patients were monitored for respiration rate with acoustic respiration rate monitoring, capnometry and impedance pneumography and values were compared to the manual counting of breaths by observation of chest wall movements. Additionally, when any respiration rate monitor indicated a cessation of breathing for 30 seconds or greater, the presumed apnea was confirmed by direct observation of the patient for absence of chest wall movements. Measurements and Main Results: Bias and precision for respiration rate measurement was $0 \pm 1.0 \mathrm{bpm}$ for acoustic monitoring, $4.8 \pm 15.1 \mathrm{bpm}$ for capnometry and $0.4 \pm 5.9 \mathrm{bpm}$ for impedance pneumography. Sensitivity and specificity for detection of apnea was $73 \%$ and $93 \%$ for acoustic monitoring, $73 \%$ and $12 \%$ for capnometry and $45 \%$ and $93 \%$ for impedance pneumography. Conclusions: Acoustic respiration rate monitoring was found to be accurate for assessment of respiration rate and to have similar or better sensitivity and specificity for detection of apnea compared to capnometry and impedance pneumography in the setting of upper GI endoscopy.
\end{abstract}

Keywords: Capnography; Endoscopy; Monitoring; Physiologic Respiratory Rate

\section{Introduction}

Apnea and respiratory depression are common occurrences during upper GI endoscopy under propofol anesthesia [1]. There are very few studies looking at the incidence of apnea during GI endoscopy but many cases of dangerous apnea reported in GI endoscopy literature. The incidence of apnea in patients undergoing complex upper GI endoscopic procedures under conscious sedation was found to be $57 \%$ in one study [2]. In another study, hypoxemia was the most commonly reported adverse event, occurring in approximately $13 \%$ of advanced endoscopic procedures with propofol [3]. The American Society of Anesthesiologists mandates the monitoring of respiration by measuring end tidal carbon dioxide during procedural sedation and anesthesia [4]. However capnometry $\left(\mathrm{EtCO}_{2}\right)$ can be unreliable in this setting. In patients who are unintubated, the sample point is normally at the nostrils; however these patients could be breathing via the mouth as it is open for upper GI endoscopy. Additionally, $\mathrm{EtCO}_{2}$ can report hypopnea as apnea depending on the device settings. Moreover, due to inadequate sampling, measured maximum carbon dioxide $\left(\mathrm{CO}_{2}\right)$ values are likely to be less than the true end tidal values. If a nasal trumpet is used to administer high flow oxygen with an endotracheal tube adapter attached to a Mapleson circuit, as is the practice in our institution (Figure 1), the sampling devices becomes even less useful.

The other available methods for monitoring respiration rate during upper GI endoscopy also have significant limitations. Pulse oximetry, for example, was found to detect only $50 \%$ of apnea or inadequate ventilation events 


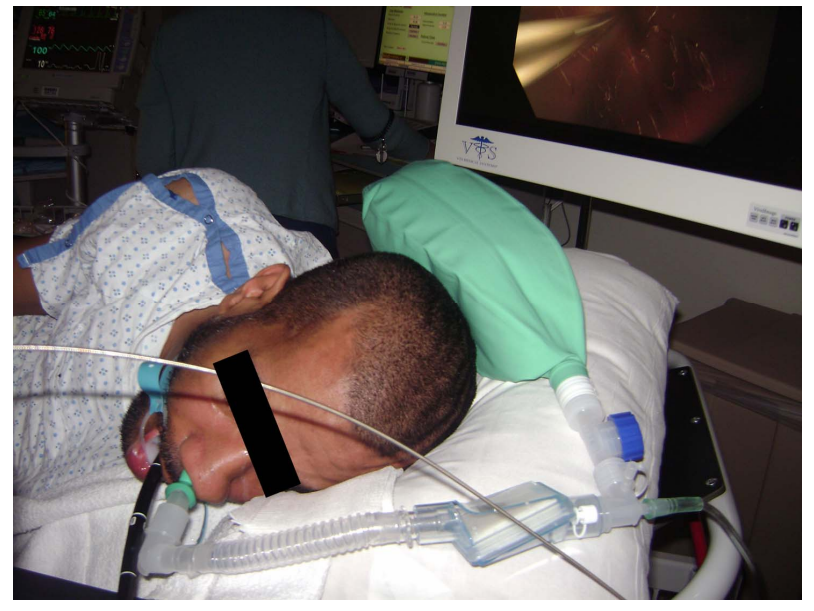

Figure 1. Nasal trumpet with an endotracheal tube adapter attached to a Mapleson circuit.

during therapeutic upper endoscopy [2]. Additionally, pulse oximetry $\left(\mathrm{SpO}_{2}\right)$ is a lagging indicator of hypoventilation especially in patients receiving supplemental oxygen [5]. Thus, valuable time could be lost in implementing corrective measures, thereby risking severe hypoxemia and possible cardiac arrest. Impedance pneumography, on the other hand, requires significant chest wall movement to record respiration and continues to show active respiratory activity in patients breathing against a partial or completely closed glottis. Patient movement artifacts (to facilitate the gastroscope insertion, for example) can also adversely affect its accuracy.

At the Hospital of the University of Pennsylvania, roughly $12,000 \mathrm{GI}$ endoscopies are performed each year. Of these, $30 \%-50 \%$ are done under propofol anesthesia as they are considered high risk and hence unsuitable for nurse administered conscious sedation. Typically the airway is not secured with an endotracheal tube or a Laryngeal Mask Airway. Consequently the patient is breathing spontaneously with an unsecured natural airway. Currently, respiration is monitored using $\mathrm{EtCO}_{2}$ monitoring and thoracic impedance pneumography. In our experience, both these methods have low sensitivity and specificity for detection of apnea in the setting of GI endoscopy anesthesia. It is clear, therefore, that an accurate and reliable respiration rate monitor for patients undergoing upper GI endoscopy is desirable. There are no studies examining the use of an acoustic respiration rate monitor in anesthetized patients undergoing GI endoscopy. Acoustic respiration rate monitoring (RRa) with Pulse CO-Oximetry uses a piezoelectric acoustic sensor placed on the neck to detect breath sounds from which respiration rate is derived. Our objective was to compare Rainbow acoustic monitoring $(\mathrm{RRa})$ and standard of care respiration rate monitors $\left(\mathrm{EtCO}_{2}\right.$ and impedance pneumography) with the manual counting of breaths and direct observation for accuracy of respiration rate and detection of apnea in patients undergoing advanced GI endoscopic procedures.

\section{Material and Methods}

After obtaining Institutional Review Board approval and written informed consent of subjects, we prospectively evaluated the accuracy of respiration rate estimations by $\mathrm{RRa}, \mathrm{EtCO}_{2}$ and impedance pheumography compared to the manual counting of breaths by observation of chest wall movements, the reference method, in a convenience sample of patients undergoing advanced endoscopic procedures. We also evaluated the ability of the three methods to detect apnea by confirming any presumed apneic event (cessation of breathing for at least 30 seconds as reported by one device) with direct observation of the patient for chest wall movements and no signs of obstructed breathing. Endoscopic procedures included endoscopic retrograde cholangiopancreatogram (ERCP), esophagogastroduodenoscopy (EGD) with ultrasound, enteroscopy, EGD for therapeutic intervention e.g., EMR, Halo 360, polyps and other EGD procedures likely to last over 30 minutes.

Patients were excluded from the study if they were unable to provide informed consent, had allergy to propofol, fentanyl or remifentanil, or required endotracheal intubation. After a thorough medical history and physical examination, sensors and cannulas connected to the appropriate monitors were placed on the patients (for $\mathrm{EtCO}_{2}$, a nasal cannula connect to a Microsteam Smart CapnoLine (R) Oridion, for impedance pneumography, sensors connected to AG-920RA bedside monitor, Nihon Koden; and for RRa, a RAS 125 , revision C adult acoustic respiration rate sensor, connected to a RAD-87 Pulse CO-Oximeter, software version 1402, 7713, Masimo Corp.) The nurse anesthetist and/or the anesthesiologist observed the digital display of respiration rate and waveform of the $\mathrm{EtCO}_{2}$ and impedance pneumogram for the occurrence of apnea while being blinded to the RRa monitor. A research technician observed the RRa monitor for the occurrence of apnea. This study was designed to detect either central or obstructive apneas as both are defined as a cessation in breathing (respiration rate of zero). For the purposes of this study, apnea was defined as a respiration rate of zero for at least 30 seconds for all devices. We used a $>30$ second threshold for apnea because this definition has been used in other studies (REF) and because all three monitors could be set to alarm after 30 seconds of a zero respiration rate. All patients were preoxygenated for at least 5 vital capacity breaths before induction with propofol (after fentanyl administration). Induction was carried out with 0.25 to $0.5 \mathrm{mcg}$ of fentanyl followed by a bolus of propofol ( $1-2 \mathrm{mg}$ per $\mathrm{kg})$ 
(proceeded by lidocaine $20-40 \mathrm{mg}$ )) to be titrated to the effect. This was followed by propofol infusion of 50 $200 \mathrm{mcg}$ per kg per min. Intermittent boluses of $20-50$ $\mathrm{mg}$ propofol were administered as necessary. In patients receiving remifetanil, fentanyl was avoided. Instead after a bolus of propofol, they were given an infusion of propofol freshly mixed with remifentanil at $5 \mathrm{mcg} / \mathrm{ml}$ concentration. This was infused at $50-120 \mathrm{mcg}$ per $\mathrm{kg}$ per $\min$. This method of anaesthesia induction and maintenance is the standard practice in endoscopy.

False alarm rate, sensitivity and specificity, and positive and negative predictive values for the detection of apnea by the three devices $\left(\mathrm{EtCO}_{2}\right.$, impedance pneumography and RRa monitoring) were determined. A true apnea (true positive, TP) was defined as an apnea event detected by any method which was confirmed by the anesthesia provider by observation of the patient for chest wall movements, and/or signs of obstructive breathing such as snoring, choking, snorting, or gasping. All presumed apneic events were confirmed to be true or determined to be false by direct observation of the patient by the anesthesia provider. A false alarm (false positive, FP) was defined as a situation where a device reported zero respiration for at least 30 seconds, while direct observation by the anesthesia provider indicated valid respiration rates and breathing. A true negative (TN) was defined as the times when a device reported normal breathing which was confirmed by direct observation of the patient but one or two of the other devices showed a cessation of breathing for $\geq 30 \mathrm{sec}$. Therefore the total number of false alarms by all devices made up the number of true negatives. A false negative (FN) was defined as the number of times the device reported a respiration rate above zero when one or two of the other devices indicated a zero respiration rate for at least 30 seconds which was confirmed by direct observation. Sensitivity was defined as the number of times the device detected a true apnea divided by the number of true apneas, plus the total number of true apneas missed (TP/[TP+FN]). Specificity was defined as the number of true negatives divided by the number of true negative plus the number of false alarms, (TN/[TN+FP]. Positive predictive value (PPV) was calculated as the number of true positives divided by the number of true positives plus the number of false positives for each device and negative predictive value (NPV) was calculated as the number of true negatives divided by the number of true negative plus the number of false negatives. 95\% confidence intervals (CI) were calculated for sensitivity, specificity positive and negative predictive values. Apnea was treated or watched carefully as appropriate.

Periodically during the study and when the study technician had good visibility of the patient's chest move- ments, respiration rate was assessed by visual inspection of chest wall movements for 30 seconds and the respiration rate for each device was recorded for that period. These data sets were used to calculate the bias (the mean difference of the respiration rate determined by the test device and reference method) and precision (one standard deviation of the bias) for each method in breaths per minute (bpm). A t-test was used to determine if differences in bias and precision between devices were significant, with $p>0.05$ considered significant.

To assess the accuracy of respiration rate estimation by each method over the range of respiration rates observed, Bland and Altman graphs corrected for multiple measures on a single subject [6], showing bias and limits of agreement, were constructed (Excel 2010, Microsoft Corp, Redmond WA). 95\% Confidence intervals were calculated for the limits of agreement.

\section{Results}

We enrolled 101 patients in the study. Three patients were excluded due to cancelation of the procedure, scheduling conflict, and equipment malfunction leaving data from 98 patients for the analysis. Patient characteristics are displayed in Table 1. During the 98 cases, there were 124 presumed apneic events, in which at least 1 device reported zero respiration for 30 seconds or longer (Table 2). Of these, 113 were false alarms (not corroborated by the direct observation). These 113 false alarm events made up the "true negatives" to which each device was compared. $\mathrm{EtCO}_{2}$ showed the highest incidence of false alarms (100) compared to impedance pneumogram (8) and the RRa monitor (8). There were 11 true apneic events. Sensitivity and specificity for detection of apnea was $73 \%$ and $93 \%$ for RRa monitoring, $73 \%$ and $12 \%$ for $\mathrm{EtCO}_{2}$ and $45 / \%$ and $93 \%$ for impedance pneumography. The positive and negative predictive values for the three devices were $50 \%$ and $97 \%$ for RRa monitoring, $7.4 \%$ and $81 \%$ for $\mathrm{EtCO}_{2}$ and $38 \%$ and $95 \%$ for impedance pneumography (Table 1). To assess bias and precision of respiratory rate estimation by each method compared to

Table 1. Patient characteristics.

\begin{tabular}{ccc}
\hline & $\mathrm{N}(\%)$ or mean $(\mathrm{SD})$ & Range \\
\hline Sex (male) & $64(65.3)$ & \\
Age (in years) & $58.7(15.3)$ & $23-91$ \\
Body mass index & $26.8(5.6)$ & $14.2-44.8$ \\
Body mass index & & \\
$<18.5$ & $6(6.1)$ & \\
$18.5-24.9$ & $32(32.7)$ & \\
$25.0-29.9$ & $32(32.7)$ & \\
$\geq 30.0$ & $26(26.5)$ & \\
\hline
\end{tabular}


Table 2. Comparison of three monitors for: a) Detection of apnea compared to the reference method of direct clinical observation of patients and; b) Accuracy of respiration rate measurement compared to the manual counting of breaths, in patients during upper GI endoscopy.

\begin{tabular}{|c|c|c|c|c|}
\hline A & Clinical observation & RRa monitoring & Capnometry & Impedance pneumography \\
\hline True positives & 11 & 8 & 8 & 5 \\
\hline True negatives & 113 & 105 & 13 & 105 \\
\hline False positives & na & 8 & 100 & 8 \\
\hline False negatives & na & 3 & 3 & 6 \\
\hline Sensitivity $=\mathrm{TP} /[\mathrm{TP}+\mathrm{FN}][\mathrm{CI}], \%$ & na & $73[39$ - 93.9] & $73[39$ - 93.9] & $45[16.8-76.6]$ \\
\hline Specificity $=\mathrm{TN} /[\mathrm{TN}+\mathrm{FP}][\mathrm{CI}], \%$ & $n a$ & $93[86.5$ - 96.9] & $12[6.3-18.9]$ & $93[86.5$ - 96.9] \\
\hline Positive predictive value $=(\mathrm{TP} /[\mathrm{TP}+\mathrm{FP}])[\mathrm{CI}], \%$ & na & $50[24-75]$ & $7.4[3.3-14]$ & $38[13-68]$ \\
\hline Negative predictive value $=(\mathrm{TN} /[\mathrm{TN}+\mathrm{FN}])[\mathrm{CI}], \%$ & na & $97[92.1-99.4]$ & $81[54.3-95.6]$ & $95[87.0-98.0]$ \\
\hline \multicolumn{5}{|l|}{$\mathrm{b}$} \\
\hline Respiration rate bias $+/-$ precision $[\mathrm{CI}](\mathrm{bpm})$ & na & $0+/-1.0^{*}[-0.7-0.7]$ & $4.8+/-15.1[2.0-7.6]$ & $0.4+/-5.9^{*}[-0.7-1.5]$ \\
\hline Respiration rate $95 \%$ limits of agreement (bpm) & na & $-1.9-1.9$ & $-24.8-34.5$ & $-11.1-11.9$ \\
\hline
\end{tabular}

$\mathrm{Na}=$ not applicable; $\mathrm{TP}=$ true positive; $\mathrm{FN}=$ false negative; $\mathrm{TN}=$ true negative; $\mathrm{FP}=$ false positive; $\mathrm{CI}=95 \%$ confidence interval. ${ }^{*}$ Difference was significant compared to capnometry, $\mathrm{p} \leq 0.05$.

visual inspection of chest wall movement, manual assessment of respiration rate was done once in 51 patients, twice in 27 patients, three times in 3 patients and was not conducted in 18 patients so there was a total of 114 assessments conducted in 81 patients. The range of manual respiration rate values observed during these assessment periods was 0 to $33 \mathrm{bpm}$. Bias and precision for respiration rate were $0+/-1.0 \mathrm{bpm}$ for RRa monitoring, $4.8+/-$ $15.1 \mathrm{bpm}$ for $\mathrm{EtCO}_{2}$ and $0.1+/-5.9 \mathrm{bpm}$ for impedance pneumography. The differences in bias for RRa monitoring and capnometry $(p=0.000)$ and impedance pneumography and capnometry $(p=0.003)$ were significant.

Bland and Altman plots showed limits of agreement of -1.9 (CI $-2.2--1.6$ ) to $1.9 \mathrm{bpm}(\mathrm{CI} 1.6-2.2)$ for $\mathrm{RRa}$ monitoring, $-24.8(\mathrm{CI}-29.5--20.0)$ to $34.5 \mathrm{bpm}(\mathrm{CI}$ 29.7 - 39.2) for $\mathrm{EtCO}_{2}$ and $-11.1(\mathrm{CI}-12.9-9.3)$ to $11.9 \mathrm{bpm}$ (CI 10.1 - 13.7) for impedance pneumography (Figure 2).

\section{Discussion}

Our study shows that both RRa monitoring and impedance pneumography provided more accurate respiration rate values compared to $\mathrm{EtCO}_{2}$, a standard of care monitor for estimation of respiration rate during complex, upper GI endoscopy. Further, RRa monitoring had fewer false alarms and similar or better sensitivity and specificity for the detection of apnea than the other two methods. We observed 11 true apneic events in 98 patients with some patients experiencing more than one true apnea. The incidence of true apnea in our study was signifi- cantly lower than what has been reported by other studies for upper GI endoscopy [2] although we did observed a large number of "presumed apneic events". It is possible therefore that the higher incidence of apnea reported in other studies may be due to counting of false positives by capnometry or impedance pneumography. It is also important to note that, due to our study design, we did not record apneas shorter than 30 seconds in duration. Other studies that considered shorter periods of cessation of breathing as apnea would likely have a higher frequency of these events.

Because any patient who has detectable carbon dioxide present at the nasal cannula sample inlet should be breathing, we expected the $\mathrm{EtCO}_{2}$ monitor to have the highest specificity in apnea detection. We were surprised to see three instances when $\mathrm{EtCO}_{2}$ reported a respiration rate even though observation confirmed the patient was apneic. These false negatives could be due to equipment malfunctioned but we believe it is more likely that the carbon dioxide present in the sample line to facilitate the endoscope was detected and displayed as true respiration. Although air is more commonly used in upper GI endoscopes, carbon dioxide is used in our hospital for ERCPS and enteroscopies. This also explains our observation of very high carbon dioxide values displayed by the monitor sometimes up to $110 \mathrm{~mm} \mathrm{Hg}$.

Although impedance pneumography was more accurate than $\mathrm{EtCO}_{2}$ for estimation of respiration rate, this method was also plagued with significant limitations for use in this setting. In 6 instances impedance pneumography 


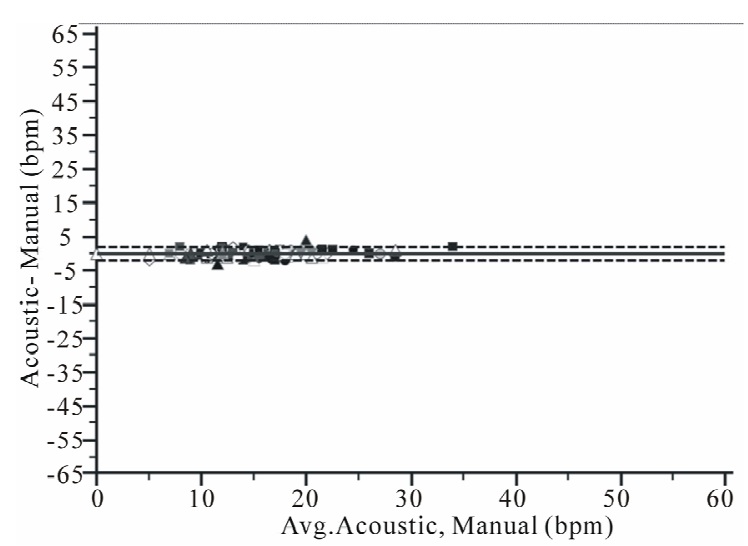

(a)

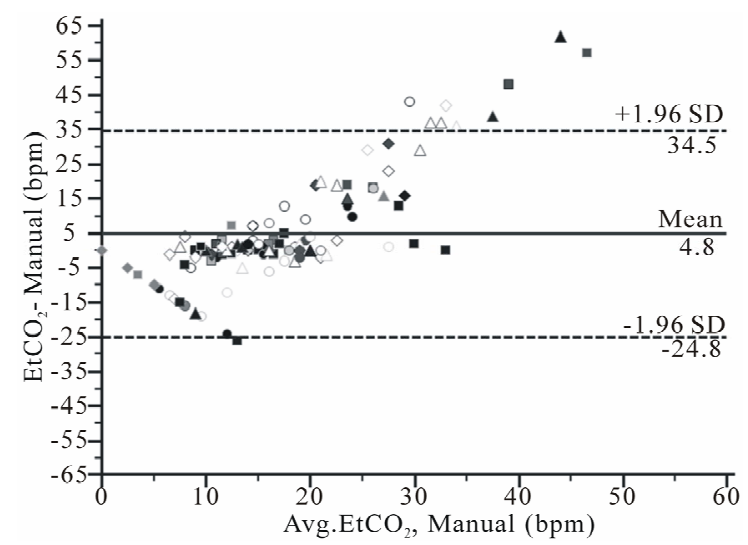

(b)

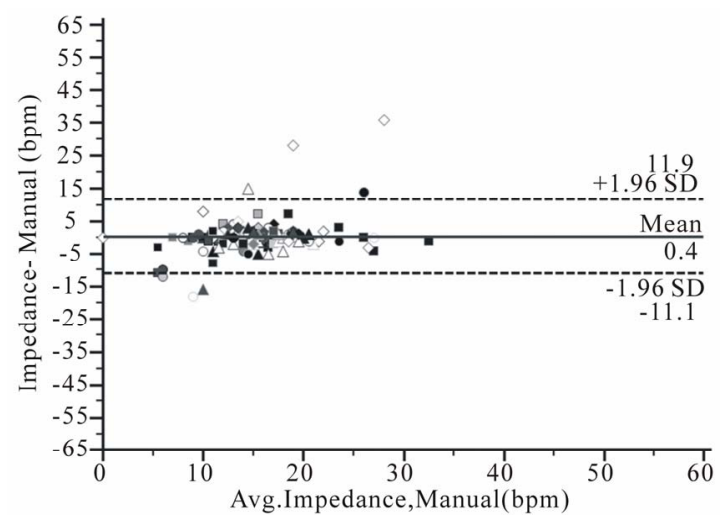

(c)

Figure 2. Bland and Altman plots for respiration rate measurement by a) RRa monitoring (RRa); b) Capnometry $\left(\mathrm{EtCO}_{2}\right)$ and c) Impedance pneuography (impedance), compare to manual counting of breaths (manual) during upper GI endoscopy from 114 comparisons in 81 patient, showing bias (solid line) and limits of agreement (2 SD) (dotted line).

detected breathing during a true apnea. Especially in ERCP procedures done in the prone position in an intubated patient, impedance pneumography detected respiration in the presence of obstructive apnea, due to the patient breathing against a closed glottis, thereby delay- ing detection of true apnea. Additionally, impedance pneumography tended to overestimate the respiration rate (bias of $4.8 \mathrm{bpm}$ ) which may have been a result of cardiogenic oscillations being detected as breaths. This limitation has been noted in other studies [7]. For all three monitors however, our observations were that events such as patient repositioning, sample line movement and insertion of a bite block could impact the accuracy.

None of the previous published reports on the accuracy of RRa monitoring, most of which have appeared only as conference abstracts, were conducted during procedural anesthesia $[8,9]$. Only one study reported on the detection of cessation of breathing [9]. A study conducted in 34 adult patients in the post anesthesia care unit found RRa monitoring to have superior sensitivity and specificity for detection of respiratory pause events (defined as the cessation of breathing for $\geq 30 \mathrm{sec}$.) compared to capnometry. In our study, both RRa monitoring and impedance pneumography had far fewer false alarms than $\mathrm{EtCO}_{2}$ (8 and 8 versus 100) and thus much better specificity. RRa monitoring, however, was able to detect more true apneas compared to impedance pneumography ( 8 versus 5 out of 11 ) but a larger sample size is needed to determine if this difference is significant. RRa monitoring therefore, proved to be an accurate monitoring solution with apnea detection which was similar or better than other methods in this setting while being reliable and easy to use.

A limitation of our study is the few number of true apneas experienced by our cohort and this may be due in part to only counting apneas of 30 seconds or greater. A larger study with a higher incidence of apneas is required to determine if the trends observed in our study can be generalized to most patients undergoing upper GI endoscopy. Also, due to lack of any standard in this setting, we used manual counting of breaths for the reference respiration rate but this method can also be subject to errors [10]. We also used direct observation of the patient by the anesthesia provider to detect apnea and believe this is the most accurate method for apnea detection, but it is not practical for the standard care.

With these concerns in mind it may be precipitous to recommend RRa monitoring as the standard respiration monitor in patients undergoing upper GI endoscopy. Likewise, however, it is equally precipitous to recommend $\mathrm{EtCO}_{2}$ as a standard monitor for procedures using anesthesia and conscious sedation. Despite the ASA recommendation, we perceive reluctance by care givers to use $\mathrm{EtCO}_{2}$ during procedural sedation and anesthesia due to the many false apnea alarms and falsely high $\mathrm{EtCO}_{2}$ values when carbon dioxide is used for enteroscopy and ERCP. The ASA mandate to use $\mathrm{EtCO}_{2}$ in this setting, therefore, should be reexamined in light of the comer- 
cialization of new and perhaps superior monitoring technologies.

\section{Conclusion}

In adult patients undergoing advanced GI endoscopic procedures, RRa respiration rate monitoring was more accurate and provided apnea detection which was similar or better than $\mathrm{EtCO}_{2}$ or impedance pneumography, two standard of care monitors in the endoscopy suite. Further studies are required to validate acoustic respiration rate monitoring in other patient populations.

\section{REFERENCES}

[1] J. J. Vargo, G. Zuccaro Jr., J. A. Dumot, S. S. Shay, D. L. Conwell and J. B. Morrow, "Gastroenterologist-Administered Propofol for Therapeutic Upper Endoscopy with Graphic Assessment of Respiratory Activity: A Case Series," Gastrointestinal Endoscopy, Vol. 52, No. 2, 2000, pp. 250-255.

[2] J. J. Vargo, G. Zuccaro Jr., J. A. Dumot, S. S. Shay, D. L. Conwell and J. B. Morrow, "Automated Graphic Assessment of Respiratory Activity Is Superior to Pulse Oximetry and Visual Assessment for the Detection of Early Respiratory Depression during Therapeutic Upper Endoscopy," Gastrointestinal Endoscopy, Vol. 55, No. 7, 2002, pp. 826-831.

[3] G. A. Cote, R. M. Hovis, M. A. Ansstas, L. Waldbaum, A R. R. zar, D. S. Early, S. A. Edmundowicz, D. K. Mullady and S. S. Jonnalagadda, "Incidence of Sedation-Related Complications with Propofol Use during Advanced Endoscopic Procedures," Clinical Gastroenterology and Hepatology, Vol. 8, No. 2, 2009, pp. 137-142.
[4] American Society of Anesthesiologists, "Standard for Basic Anesthetic Monitoring," American Society of Anesthesiologists, Inc., Park Ridge, 2011.

[5] E. S. Fu, J. B. Downs, J. W. Schweiger, R. V. Miguel and R. A. Smith, "Supplemental Oxygen Impairs Detection of Hypoventilation by Pulse Oximetry," Chest, Vol. 126, No. 5, 2004, pp. 1552-1558.

[6] J. M. Bland and D. G. Altman, "Agreement between Methods of Measurement with Multiple Observations per Individual," Journal of Biopharmaceutical Statistics, Vol. 17, No. 4, 2007, pp. 571-582. doi:10.1080/10543400701329422

[7] K. P. Cohen, W. M. Ladd, D. M. Beams, W. S. Sheers, R. G. Radwin, W. J. Tompkins and J. G. Webster, "Comparison of Impedance and Inductance Ventilation Sensors on Adults during Breathing, Motion, and Simulated Airway Obstruction," IEEE Transactions on Biomedical Engineering, Vol. 44, No. 7, 1997, pp. 555-566. doi:10.1109/10.594896

[8] O. Mimoz, T. Benard, A. Gaucher, D. Frasca and B. Debaene, "Accuracy of Respiratory Rate Monitoring Using a Non-Invasive Acoustic Method after General Anaesthesia," British Journal of Anaesthesia, Vol. 108, No. 5, 2012, pp. 872-875. doi:10.1093/bja/aer510

[9] M. A. Ramsay and M. Usman, "Accuracy of Respiration Rate and Detection of Respiratory Pause by Acoustic Respiratory Monitoring in the PACU," The 65th Annual Post Graduate Assembly in Anesthesiology, New York, 9-11 December 2011, p. 9137.

[10] B. J. Semmes, M. J. Tobin, J. V. Snyder and A. Grenvik, "Subjective and Objective Measurement of Tidal Volume in Critically Ill Patients," Chest, Vol. 87, No. 5, 1985, pp. 577-579. doi:10.1378/chest.87.5.577 